\title{
Musical Minds: Attentional Blink Reveals Modality-Specific Restrictions
}

\author{
Sander Martens $\mathbf{s}^{1,2 *}$, Stefan M. Wierda ${ }^{1,2,3}$, Mathijs Dun ${ }^{1}$, Michal de Vries ${ }^{1,3}$, \\ Henderikus G. O. M. Smid ${ }^{1}$ \\ 1 Neuroimaging Center, University of Groningen, Groningen, the Netherlands, 2 Department of \\ Neuroscience, University Medical Center Groningen, Groningen, the Netherlands, 3 Institute of Artificial \\ Intelligence, University of Groningen, Groningen, the Netherlands \\ *s.martens@umcg.nl
}

\section{Abstract}

\section{Background}

Formal musical training is known to have positive effects on attentional and executive functioning, processing speed, and working memory. Consequently, one may expect to find differences in the dynamics of temporal attention between musicians and non-musicians.

\section{G OPEnACCESS}

Citation: Martens S, Wierda SM, Dun M, de Vries M, Smid HGOM (2015) Musical Minds: Attentional Blink Reveals Modality-Specific Restrictions. PLoS ONE 10(2): e0118294. doi:10.1371/journal.pone.0118294

Academic Editor: Jan de Fockert, Goldsmiths University of London, UK, UNITED KINGDOM

Received: July 7, 2014

Accepted: January 13, 2015

Published: February 25, 2015

Copyright: $\odot 2015$ Martens et al. This is an open access article distributed under the terms of the Creative Commons Attribution License, which permits unrestricted use, distribution, and reproduction in any medium, provided the original author and source are credited.

Data Availability Statement: All relevant data are within the paper and its Supporting Information files.

Funding: This research was supported by a grant of the Behavioural and Cognitive Neurosciences (BCN) Research School. The funders had no role in study design, data collection and analysis, decision to publish, or preparation of the manuscript. Here we address the question whether that is indeed the case, and whether any beneficial effects of musical training on temporal attention are modality specific or generalize across sensory modalities.

\section{Methodology/Principal Findings}

When two targets are presented in close temporal succession, most people fail to report the second target, a phenomenon known as the attentional blink (AB). We measured and compared $A B$ magnitude for musicians and non-musicians using auditory or visually presented letters and digits. Relative to non-musicians, the auditory $A B$ was both attenuated and delayed in musicians, whereas the visual $A B$ was larger. Non-musicians with a large auditory $A B$ tended to show a large visual $A B$. However, neither a positive nor negative correlation was found in musicians, suggesting that at least in musicians, attentional restrictions within each modality are completely separate.

\section{Conclusion/Significance}

$A B$ magnitude within one modality can generalize to another modality, but this turns out not to be the case for every individual. Formal musical training seems to have a domain-general, but modality-specific beneficial effect on selective attention. The results fit with the idea that a major source of attentional restriction as reflected in the AB lies in modality-specific, independent sensory systems rather than a central amodal system. The findings demonstrate that individual differences in $A B$ magnitude can provide important information about the modular structure of human cognition.

Competing Interests: The authors have declared that no competing interests exist. 


\section{Introduction}

"Music is the art of thinking with sounds", according to the French music scholar Jules Combarieu (1859-1915). Being an important part of human culture, everyday exposure to music teaches most children basic musical competence, enabling them for instance to tap and dance to music, detect wrong notes, remember and reproduce familiar tunes and rhythms, and feel the emotions expressed in music [1]. On top of the common experience of listening to music, it has been shown that formal musical training can have a number of domain-specific effects on music perception [2-7] and can alter structure and function of localized brain areas [8-11]. Although there has been some controversy regarding the question whether formal musical experience can also have domain-general effects (i.e., effects that are not specific to the perception of music) [12], several studies have reported that explicit musical instruction benefits a range of domain-general abilities such as pre-reading and reading ability, mathematical and spatial abilities, creativity, and general intelligence [13-16].

Germane to the current study, beneficial effects on attentional and executive functioning, processing speed, and working memory have also been observed [17-20]. For instance, a recent study by Zuk et al. [20] found that musicians demonstrate enhanced performance on several constructs of cognitive flexibility, such as verbal fluency (the ability to name as many objects that match a certain criterion (e.g. animals) within 60 seconds), design fluency (the ability to connect a set series of dots to make as many different designs as possible within 60 seconds), and the Trail Making test (a measure of visual attention and task switching efficiency). In addition, they reported that musically trained children show increased brain activity in parietal areas and the right ventrolateral prefrontal cortex (VLPFC) when involved in rule representation and task-switching.

Interestingly, the same cognitive processes and associated brain areas have been suggested to play a crucial role in the Attentional Blink (AB) phenomenon [21]. The AB is a deficit in reporting the second of two targets when presented in close temporal succession. Being a central topic in attention research for more than two decades, it has proven to be a powerful tool to measure the temporal dynamics of attention [21-24]. As the $\mathrm{AB}$ can be obtained using a variety of stimuli and task conditions, it is thought to reflect a very general property of perceptual awareness [21]. However, while the $A B$ is considered to arise from a fundamental restriction of attentional capacity and/or control, people differ widely in the magnitude of the $A B$, with some individuals (referred to as non-blinkers) showing little or even no $\mathrm{AB}$ [21,25-44]. When comparing these non-blinkers with strong blinkers, activation differences in parietal as well as the right VLPFC have been observed [25].

Given the spatial overlap of differential brain activity for non-blinkers (vs. blinkers) and musically trained (vs. untrained) individuals, it may be interesting to investigate the $\mathrm{AB}$ in musicians. If attentional and executive functioning, processing speed, and working memory are indeed enhanced in musicians, one would predict that they may also show a reduced $\mathrm{AB}$ effect, similar to what has also been observed in expert meditators [38] and video gamers [40]. Therefore, the primary goal of the current study was to determine whether the beneficial effect of musical training on attention and working memory is reflected in the $\mathrm{AB}$.

If, in comparison to non-musicians, an effect can indeed be observed, an intriguing subsequent question will be whether it is present within the auditory modality only, or whether the positive effect of musical training on the efficiency of selective attention carries over to both the auditory and the visual modality. Both cases would provide evidence for a domain-general effect of musical training experience on selective attention. In contrast, a modality-specific effect (i.e., auditory only) would pinpoint boundaries in and between attention and 'the art of thinking in sounds'. To test these hypotheses, two experiments were carried out using stimuli of 
equivalent difficulty that were either presented in the auditory (Experiment 1 ) or visual modality (Experiment 2). In each experiment, a group of musicians and a group of non-musicians performed an $\mathrm{AB}$ task, requiring the identification of letter targets amongst a rapid sequential stream of digit distractors.

\section{Methods}

\section{Experiment 1}

Participants

A group of 29 volunteers (aged 18-34, mean $=22.4$ years) formed the musicians group, recruited from the University of Groningen community and the Prince Claus Conservatoire. All musicians had attended lessons in playing one or more musical instruments for at least 4 years and actively played a minimum of 4 hours a week. Twenty-nine additional volunteers, recruited from the University of Groningen community (aged 18-33, mean $=22.2$ ), formed the control group ("non-musicians"). Unlike the musicians, they reported to have no noteworthy musical background.

All participants had Dutch as their native language, normal or corrected-to-normal visual acuity, normal hearing and no history of neurological problems. The Neuroimaging Center Institutional Review Board approved the experimental protocol and written consent was obtained prior to the experiment. Participants received payment of $€ 6$.

Stimuli and Apparatus

Stimuli consisted of spoken consonant letters (excluding ' $\mathrm{S}$ ' and ' $\mathrm{V}$ ') and digits (excluding ' 1 ', '5', '6', '7', and ' 9 '), which were digitally recorded and compressed to $120 \mathrm{~ms}$ duration. The stimuli were presented at approximately $83 \mathrm{~dB}$ using Sony MDR-V600 headphones. The generation of stimuli and the collection of responses were controlled by using E-prime 1.2 software [45] running under Windows XP on a PC with a $2.8-\mathrm{GHz}$ processor.

Procedure

Prior to each trial, a fixation point appeared in the middle of a 17-inch CRT monitor together with a message at the bottom, prompting participants to press the space bar to initiate the trial. When the space bar was pressed, the message disappeared immediately. The fixation cross remained on the screen for $250 \mathrm{~ms}$, followed by a sequential stream of 22 stimuli. Each item was presented for $120 \mathrm{~ms}$, with an inter stimulus interval of $10 \mathrm{~ms}$. In $80 \%$ of the trials, two target letters were embedded in the stream (dual-target trials), and in $20 \%$ of the trials, only one target was present (single-target trials). In single- and dual-target trials, T1 was always presented as the fifth item in the stream. In dual-target trials, T2 was the first, second, third, or twelfth item following T1 (i.e., T2 was presented at lag 1,2,3, or 12, respectively). Thus, the stimulus onset asynchrony (SOA) between the targets randomly varied from 130, 260, 390, to $1560 \mathrm{~ms}$. Each SOA was presented equally often. Target letters were randomly selected with the constraint that T1 and T2 were always different letters. Digit distractors were randomly selected with the constraint that no single digit was presented twice in succession. After the presentation of the stimulus stream, participants were asked to identify the presented targets, if possible, by pressing the corresponding keys on the computer keyboard. Participants were instructed to take sufficient time in making their responses to ensure that typing errors were not made. If a target was missed or absent, participants were instructed to press the space bar instead. Responses were accepted and counted correct in either order. After responses were collected, participants could initiate the next trial by pressing the space bar.

The experiment consisted of three practice blocks and two experimental blocks. In the first practice block, all 23 stimuli were presented one by one, in isolation. Participants identified each stimulus by pressing the corresponding key on a keyboard. When all stimuli had been presented 
once, stimuli that were not correctly identified were presented again in random order, until all stimuli were identified correctly. The second practice block contained 24 single-target trials, during which participants were required to identify the single target letter embedded within the stream of digit distractors. Feedback was provided at the end of each trial for $1 \mathrm{~s}$. The block was repeated as long as accuracy remained below $70 \%$ (once for $n=15$, twice for $n=3$, three times for $\mathrm{n}=3$ ). In the last practice block as well as in the experimental blocks, stimulus streams with either 1 or 2 targets were presented, as described above. The practice block consisted of 30 trials, whereas the two experimental blocks consisted of 160 trials each. Feedback was provided in the practice block only. After the first experimental block, participants were allowed to take a short break. The experiment took approximately 45 minutes to complete.

\section{Experiment 2}

To investigate whether a reduced auditory $\mathrm{AB}$ is accompanied by a reduced visual $\mathrm{AB}$, an identification task similar to the auditory $\mathrm{AB}$ task was given, but using visual stimuli, similar to $[34,35]$.

Participants

All participants from Experiment 1 were re-invited and volunteered to participate in Experiment 2. The Neuroimaging Center Institutional Review Board approved the experimental protocol and written consent was obtained prior to the experiment. Participants received payment of $€ 4$.

Stimuli and Apparatus

Stimuli consisted of consonant letters (excluding 'Q') and digits (excluding ' 0 ' and ' 1 '). They were presented in black on a white background presented in 12-point Courier New font, using the same hard- and software as in Experiment 1.

Procedure

Prior to each trial, a fixation cross was presented in the middle of the screen. When the space bar was pressed, the fixation cross disappeared immediately and was followed $100 \mathrm{~ms}$ later by the rapid serial visual presentation (RSVP) stream. Like the auditory stimulus stream in Experiment 1, the visual stream consisted of 22 stimuli, containing one target in $20 \%$ of the trials, and two targets in $80 \%$ of the trials.

In contrast to Experiment 1, distractors in the visual $A B$ task were presented for $90 \mathrm{~ms}$. Following [32,34-36], we attempted to control task difficulty, keeping mean visual T1 performance in Experiment 2 equivalent to the mean auditory T1 performance in Experiment 1, for each individual participant, by manipulating the duration of visual targets in the following way.

Each block of trials began with a target duration of $70 \mathrm{~ms}$, immediately followed by a $20-\mathrm{ms}$ mask (a digit). After the first trial, target and mask duration were variable, with target duration ranging from 20 to $80 \mathrm{~ms}$. The sum of target and mask duration was always $90 \mathrm{~ms}$, thereby keeping the interval between the onset of a target and the onset of a subsequent distractor constant. After each trial, a running average of $\mathrm{T} 1$ accuracy was calculated and compared to that individual's mean T1 accuracy in Experiment 1. Whenever mean T1 accuracy in Experiment 2 became 5\% higher than the mean T1 accuracy in Experiment 1, visual target presentation duration was decreased by $10 \mathrm{~ms}$ and mask duration was increased by $10 \mathrm{~ms}$, thereby making visual target identification more difficult. When mean visual $\mathrm{T} 1$ accuracy became $5 \%$ lower than the mean auditory T1 accuracy (in Experiment 1), visual target duration was increased by $10 \mathrm{~ms}$ and mask duration decreased by $10 \mathrm{~ms}$, thereby making visual target identification easier. The identity of a mask never corresponded with the identity of a preceding or following distractor digit.

The first target was always presented as the fifth item in the stream. T2 was the first, second, third, or twelfth item following T1 (i.e., it was presented at lag 1, 2, 3, or 12, respectively), 
resulting in SOAs of 90, 180, 270, and $1080 \mathrm{~ms}$. Note that lag 2 in the auditory experiment corresponded roughly with lag 3 in the visual experiment (SOAs of 260 and 270, respectively). Response instructions as in Experiment 1 were given.

The experiment consisted of one practice block of 30 trials and two experimental blocks of 160 trials each. After each block, participants were allowed to take a short break. The experiment took approximately 30 minutes to complete.

\section{Results and Discussion}

\section{Experiment 1}

Following [46,47], the results were analyzed using binomial mixed-effects models. Given that there are large individual differences in the $\mathrm{AB}[25]$ and that our hypothesis predicted a different number of observations per cell, mixed-effects models are preferred over methods that assume an equal number of observations per cell. The use of mixed-effects models in the field of $\mathrm{AB}$ research is relatively new, but the method is widely used in other fields, such as psycholinguistics [48], eye movement data [49], or memory research [50]. Analyses were performed using lmer functions in the lme4 package [51] for the statistical software R. Fig. 1 shows the predicted probability of correct $\mathrm{T} 1$ identification as a function of the interval between the two targets (SOA) for both groups of participants. Note that the probabilities that are depicted in the figures were predicted by the mixed-effects models, which were fit to the binomial accuracy data. Also, the error bars show the upper and lower confidence limits as estimated by the model.

A binominal mixed-effects model was fitted on T1 accuracy. SOA and group were entered as fixed factors in the model. Subject was entered as a random factor. The model explains

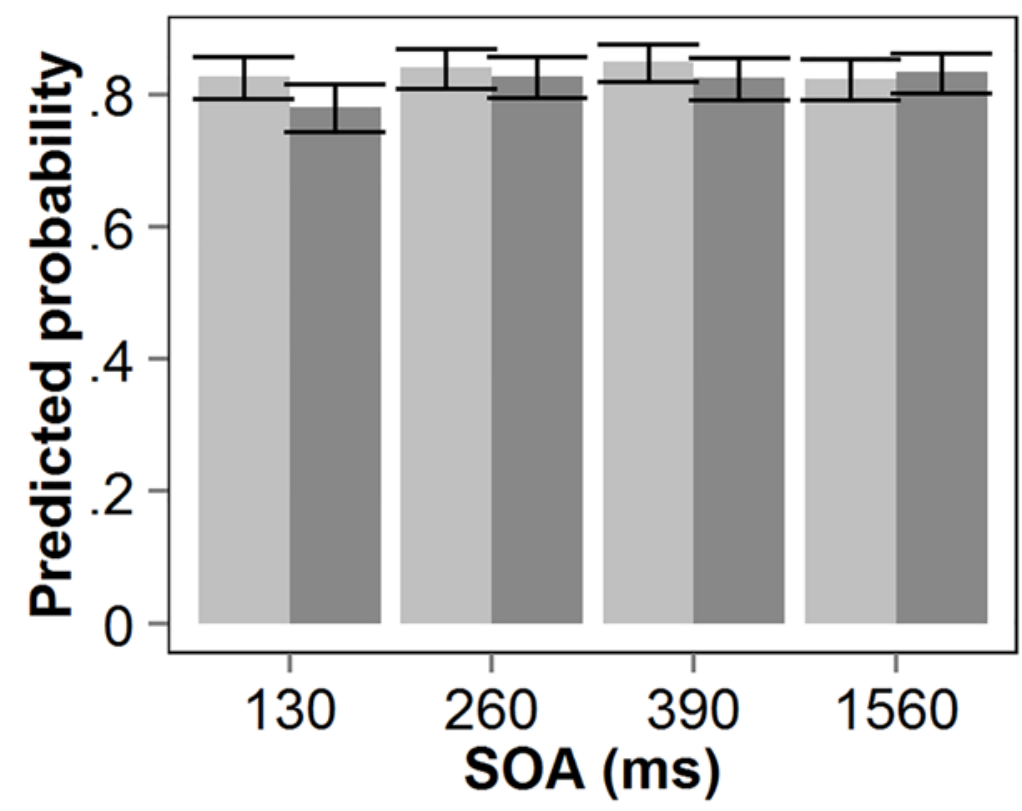

\section{Musicians Non-musicians}

Fig 1. Auditory T1 performance in Experiment 1. Predicted probability of correct T1 identification as a function of stimulus onset asynchrony (SOA) between the two auditory targets, for non-musicians and musicians. Error bars reflect upper and lower confidence limits of the model.

doi:10.1371/journal.pone.0118294.g001 
Table 1. The estimates and z-values of the mixed-effects model for T1 accuracy of the auditory AB experiment.

\begin{tabular}{|c|c|c|c|c|}
\hline & \multicolumn{4}{|c|}{ Mixed-effects model T1 } \\
\hline & Estimate & Standard Error & z-value & p-value \\
\hline Non-musicians, SOA 1560(intercept) & 1.615 & 0.112 & 14.404 & 0.000 \\
\hline Musicians & -0.066 & 0.158 & -0.415 & 0.678 \\
\hline SOA 130 & -0.344 & 0.084 & -4.112 & 0.000 \\
\hline SOA 260 & -0.042 & 0.087 & -0.479 & 0.632 \\
\hline SOA 390 & -0.060 & 0.087 & -0.695 & 0.487 \\
\hline Musicians, SOA 130 & 0.359 & 0.120 & 2.993 & 0.003 \\
\hline Musicians, SOA 260 & 0.156 & 0.123 & 1.265 & 0.206 \\
\hline Musicians, SOA 390 & 0.238 & 0.124 & 1.928 & 0.054 \\
\hline
\end{tabular}

The estimates of the fixed effects are given in log odds.

doi:10.1371/journal.pone.0118294.t001

significantly more variance compared to the null-model, $\chi^{2}(7)=28.014, p<.001$. The model's estimates, standard errors, $z$-values and $p$-values are listed in Table 1. Performance of the nonmusicians at a SOA of $1560 \mathrm{~ms}$ (i.e., lag 12) was taken as baseline. The negative estimate of SOA 130 (i.e., lag 1), reflects interference between the two targets at the first lag. In addition, there was a significant Group $\times$ SOA interaction at SOA 130 and a marginally significant Group $\times$ SOA interaction at SOA 390. The direction and size of the estimates indicate less interference between the targets at these SOAs for the musicians compared to the non-musicians. No significant difference $(p=.2)$ in single target performance was found between musicians $(84.0 \%)$ and non-musicians (82.0\%).

Fig. 2 shows the predicted probability of $\mathrm{T} 2$ given a correct $\mathrm{T} 1$ (T2|T1) as a function of the interval between the two targets (SOA) for both groups of participants. A binomial mixed-effects model was fitted on the accuracy of T2 given correct report of T1. The factors SOA and group were entered in the model as fixed factors. Subject was entered as random factor. The model explains significantly more variance compared to the null-model, $\chi^{2}(7)=56.676$, $p<.001$. Table 2 shows the model's estimates, standard errors, $z$-values and $p$-values. The model revealed an effect of SOA at 260 and $390 \mathrm{~ms}$. The negative estimates at both SOAs reflect the occurrence of an AB. A significant Group $\times$ SOA interaction was found for SOA 260 . The positive estimate indicates that musicians performed better at this SOA compared to the nonmusicians. A separate analysis of the musicians' performance only revealed a significant effect of SOA at $390 \mathrm{~ms}(z=-2.19, p=.03)$. In other words, both groups showed a significant $\mathrm{AB}$ at SOA 390. Importantly, however, the musicians' absence of an AB at SOA 260, combined with the fact that their $A B$ at SOA 390 was not as large as that of the non-musicians at SOA 260, suggest that the auditory $\mathrm{AB}$ in musicians was both delayed and attenuated.

\section{Experiment 2}

Fig. 3 shows the predicted probability of correct T1 identification as a function of the interval between the two targets (SOA) for both groups of participants.

A binominal mixed-effects model was fitted on T1 accuracy. SOA and group were entered as fixed factors in the model. Subject was entered as a random factor. The model explains significantly more variance compared to the null-model, $\chi^{2}(7)=206.23, p<.001$. The model's estimates, standard errors, $z$-values and $p$-values are listed in Table 3. The effect found for SOAs 90 and 180 reflects interference between the two targets. The lack of a significant main group effect or interaction indicates that $\mathrm{T} 1$ performance was equivalent for the two groups. 


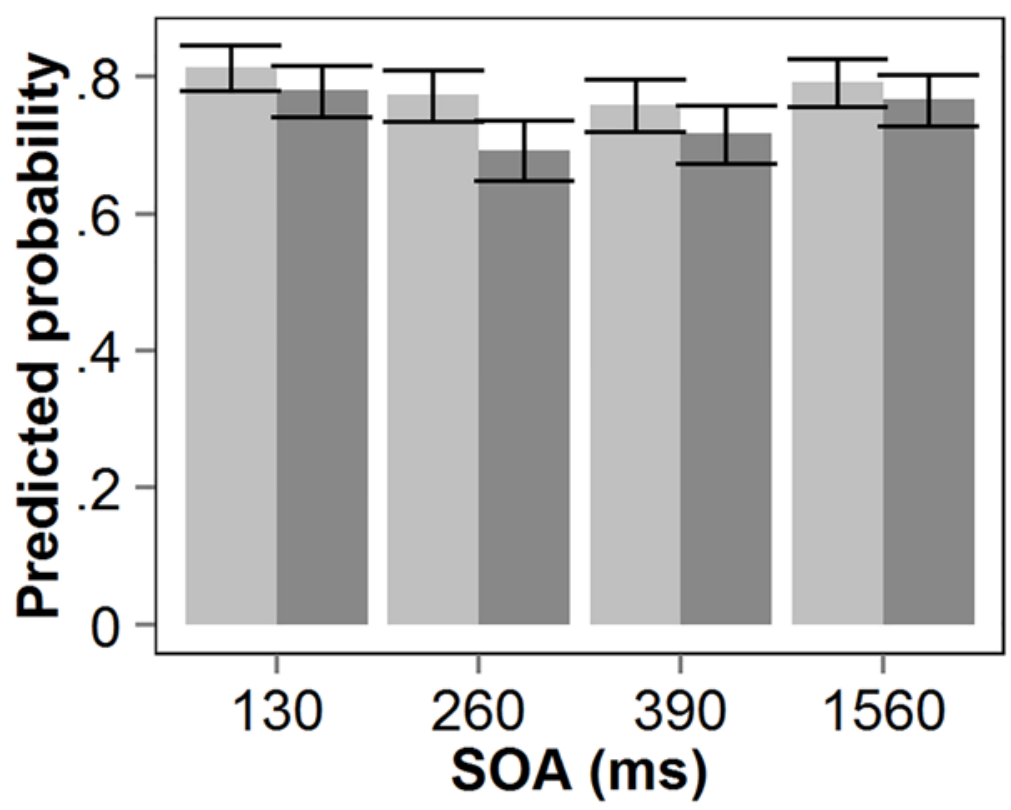

\section{Musicians Non-musicians}

Fig 2. Auditory T2|T1 performance in Experiment 1. Predicted probability of T2 given correct report of T1, as a function of stimulus onset asynchrony (SOA) between the two auditory targets, for non-musicians and musicians. Error bars reflect upper and lower confidence limits of the model.

doi:10.1371/journal.pone.0118294.g002

No significant difference $(p=.7)$ in single target performance was found between musicians (77.1\%) and non-musicians (75.8\%). Using a mixed-effect model to analyze mean target duration, calculating the $p$-value by performing 10000 Markov Chain Monte Carlo (MCMC) samplings, no significant difference was found between musicians $(71 \mathrm{~ms})$ and non-musicians (72 ms), $t=.21, p=.83$. A comparison between (dual-target) T1 performance in Experiment 1 and 2 revealed that mean auditory T1 performance $(80.3 \%)$ was significantly better $(z=-7.45$, $p<.001)$ than the mean visual T1 performance $(76.4 \%)$, but that these differences were similar for both groups ( $p s>.3$ ).

Table 2. The estimates and z-values of the mixed-effects model for T2|T1 accuracy of the auditory AB experiment.

\begin{tabular}{|c|c|c|c|c|}
\hline & \multicolumn{4}{|c|}{ Mixed-effects model T2|T1 } \\
\hline & Estimate & Standard Error & z-value & p-value \\
\hline Non-musicians, SOA 1560 (intercept) & 1.191 & 0.109 & 10.981 & 0.000 \\
\hline Musicians & 0.151 & 0.155 & 0.973 & 0.330 \\
\hline SOA 130 & 0.077 & 0.089 & 0.866 & 0.386 \\
\hline SOA 260 & -0.377 & 0.084 & -4.514 & 0.000 \\
\hline SOA 390 & -0.263 & 0.084 & -3.124 & 0.002 \\
\hline Musicians, SOA 130 & 0.059 & 0.129 & 0.457 & 0.648 \\
\hline Musicians, SOA 260 & 0.267 & 0.122 & 2.181 & 0.029 \\
\hline Musicians, SOA 390 & 0.070 & 0.577 & 2.850 & 0.564 \\
\hline
\end{tabular}

The estimates of the fixed effects are given in log odds.

doi:10.1371/journal.pone.0118294.t002 


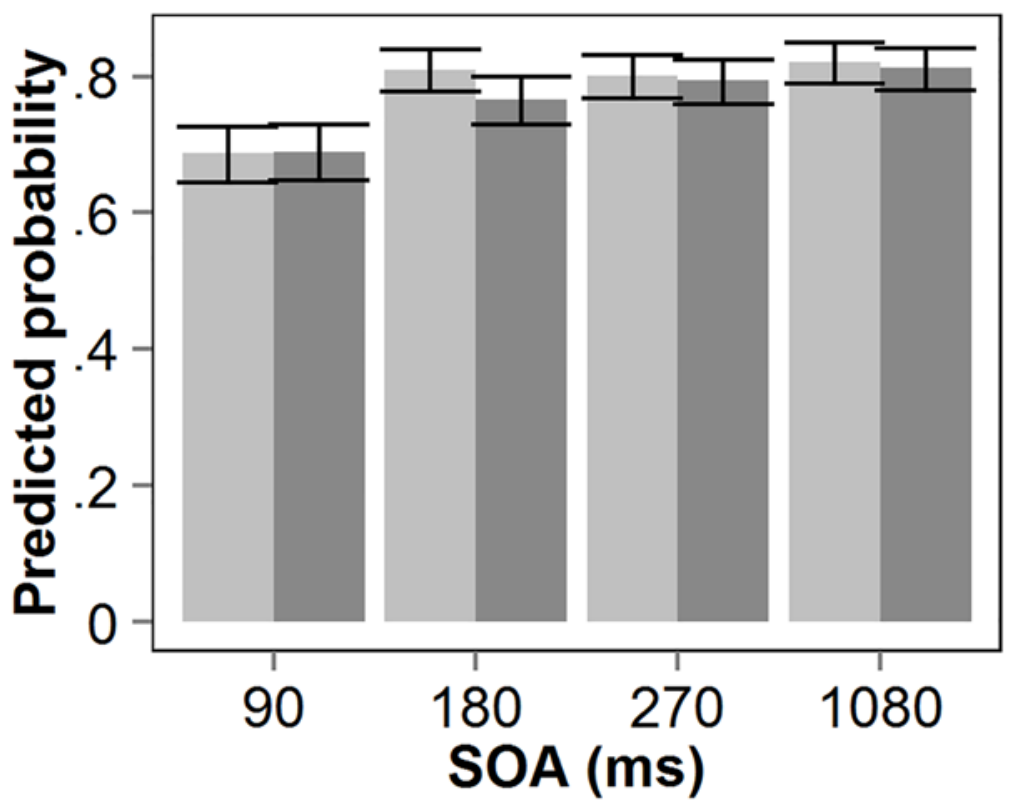

\section{Musicians Non-musicians}

Fig 3. Visual T1 performance in Experiment 2. Predicted probability of correct $\mathrm{T} 1$ identification as a function of stimulus onset asynchrony (SOA) between the two visual targets, for non-musicians and musicians. Error bars reflect upper and lower confidence limits of the model.

doi:10.1371/journal.pone.0118294.g003

Fig. 4 shows the predicted probability of $\mathrm{T} 2$ given a correct $\mathrm{T} 1$ (T2|T1) as a function of the interval between the two targets (SOA) for both groups of participants. A binomial mixedeffects model was fitted on the accuracy of $\mathrm{T} 2$ given correct report of T1. The factors SOA and group were entered in the model as fixed factors. Subject was entered as random factor. The model explains significantly more variance compared to the null-model, $\chi^{2}(7)=1089$, $p<.001$. Table 4 shows the model's estimates, standard errors, $z$-values and $p$-values. The model revealed an effect of SOA at 90, 180, and $270 \mathrm{~ms}$. The negative estimates at SOAs 180 and 270 reflect the occurrence of an $\mathrm{AB}$, whereas the positive estimate at SOA 90 reflects lag-1

Table 3. The estimates and z-values of the mixed-effects model for T1 accuracy of the visual AB experiment.

\begin{tabular}{lllr}
\hline & & \multicolumn{2}{c}{ Mixed-effects model T1 } \\
\cline { 2 - 4 } & Estimate & Standard Error & z-value \\
\hline Non-musicians, SOA 1080(intercept) & 1.464 & 0.102 & 14.303 \\
Musicians & 0.064 & 0.145 & 0.437 \\
SOA 90 & -0.667 & 0.078 & -8.528 \\
SOA 180 & -0.278 & 0.081 & -3.434 \\
SOA 270 & -0.113 & 0.083 & -1.367 \\
Musicians, SOA 90 & -0.074 & 0.111 & -0.667 \\
Musicians, SOA 180 & 0.200 & 0.117 & 0.000 \\
Musicians, SOA 270 & -0.020 & 0.118 & 0.172 \\
\hline
\end{tabular}

The estimates of the fixed effects are given in log odds.

doi:10.1371/journal.pone.0118294.t003 


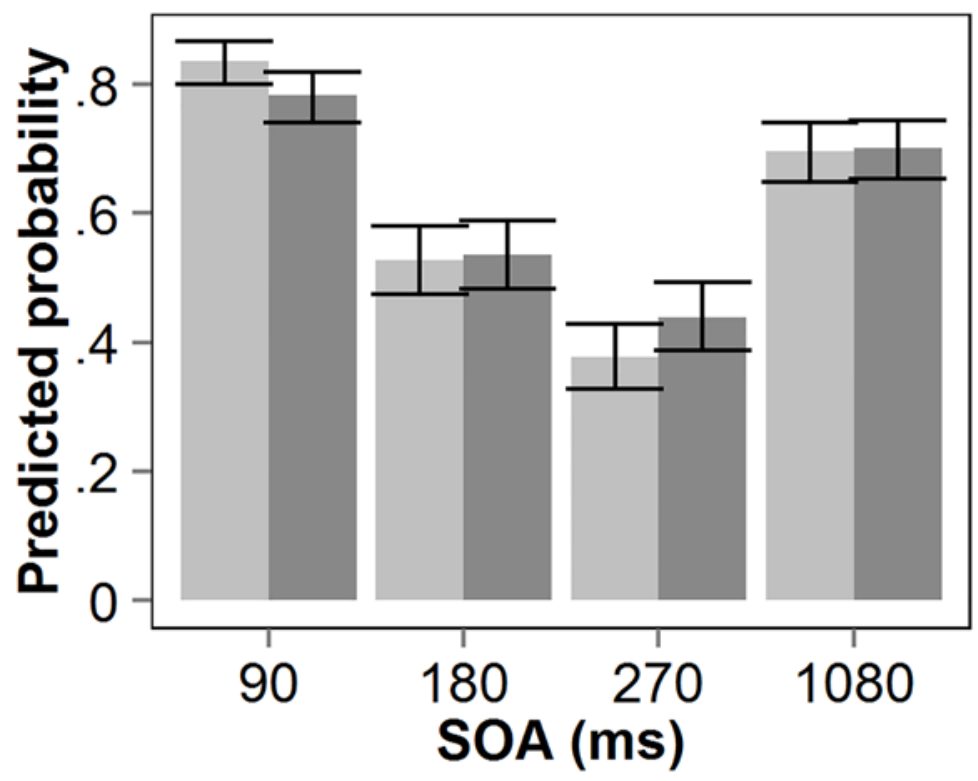

\section{Musicians Non-musicians}

Fig 4. Visual T2|T1 performance in Experiment 2. Predicted probability of T2 given correct report of T1, as a function of stimulus onset asynchrony (SOA) between the two visual targets, for non-musicians and musicians. Error bars reflect upper and lower confidence limits of the model.

doi:10.1371/journal.pone.0118294.g004

sparing [52]. Although there was no main effect of group, somewhat surprisingly, a significant Group $\times$ SOA interaction was found for SOAs 90 and 270 . The positive estimate at the SOA of $90 \mathrm{~ms}$ indicates stronger lag-1 sparing for musicians, whereas the negative estimate at the SOA of $270 \mathrm{~ms}$ reflects a greater $\mathrm{AB}$ magnitude for musicians than for non-musicians.

When mean individual $\mathrm{T} 1$ performance within the auditory modality was compared to that within the visual modality as shown in Fig. 5, significant positive Pearson product-moment

Table 4. The estimates and z-values of the mixed-effects model for T2|T1 accuracy of the visual AB experiment.

\begin{tabular}{|c|c|c|c|c|}
\hline & \multicolumn{4}{|c|}{ Mixed-effects model T2|T1 } \\
\hline & Estimate & Standard Error & z-value & p-value \\
\hline Non-musicians, SOA 1080 (intercept) & 0.850 & 0.111 & 7.658 & 0.000 \\
\hline Musicians & -0.02170 & 0.157 & -0.138 & 0.899 \\
\hline SOA 90 & 0.432 & 0.090 & 4.786 & 0.000 \\
\hline SOA 180 & -0.703 & 0.080 & -8.785 & 0.000 \\
\hline SOA 270 & -1.094 & 0.080 & -13.735 & 0.000 \\
\hline Musicians, SOA 90 & 0.372 & 0.131 & 2.829 & 0.005 \\
\hline Musicians, SOA 180 & -0.017 & 0.112 & -0.154 & 0.878 \\
\hline Musicians, SOA 270 & -0.236 & 0.113 & -2.099 & 0.036 \\
\hline
\end{tabular}

The estimates of the fixed effects are given in log odds.

doi:10.1371/journal.pone.0118294.t004 


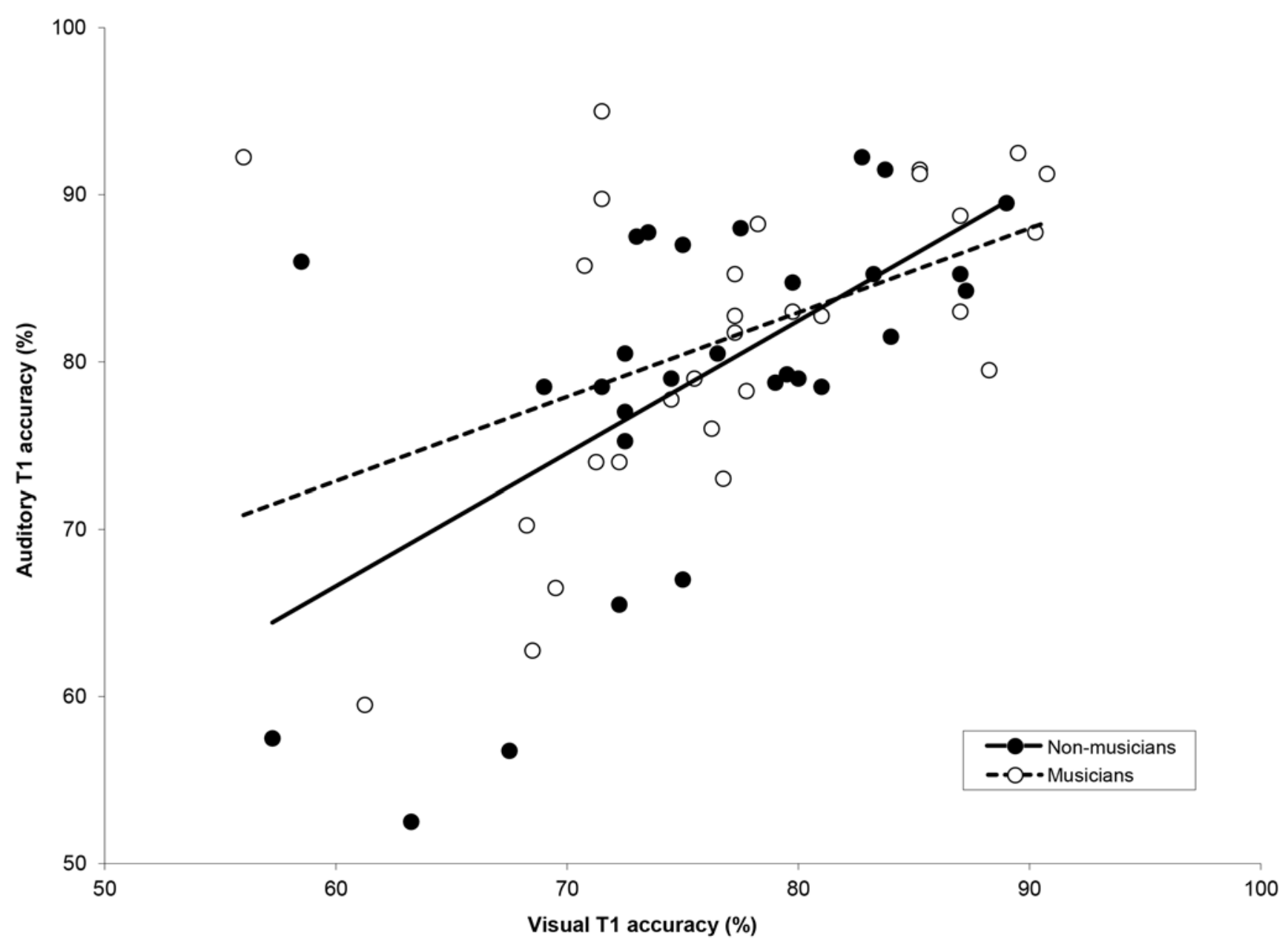

Fig 5. Mean T1 accuracy in Experiment 1 and 2. The correlation between individual T1 accuracy in the visual modality (Experiment 2) versus auditory modality (Experiment 1).

doi:10.1371/journal.pone.0118294.g005

correlations were found for each group (non-musicians: $r=.61, p<.001$; musicians: $r=.47$, $p=.01$ ). In an additional analysis shown in Fig. 6, we correlated auditory AB magnitude with visual $\mathrm{AB}$ magnitude, for musicians and non-musicians respectively. Auditory $\mathrm{AB}$ magnitude was calculated using the following formula:

$$
\left(\frac{T 2\left|T 1_{S O A 1560}-T 2\right| T 1_{S O A 260}}{T 2 \mid T 1_{S O A 1560}}\right) * 100 \%
$$

In other words, auditory $\mathrm{AB}$ magnitude reflected the decrement in performance at SOA 260 relative to the longest SOA. Visual AB magnitude was calculated similarly, based on SOAs 270 and 1080. For non-musicians, a significant positive Pearson product-moment correlation was found, $r=.39, p=.04$, indicating that non-musicians with a relatively large auditory $\mathrm{AB}$ also tend to show a large visual $\mathrm{AB}$. For musicians, however, no significant correlation between auditory and visual $\mathrm{AB}$ magnitude was found $(p=.21)$. A similar pattern of results was found when the difference in performance between the long and short SOA was taken as a measure of $\mathrm{AB}$ magnitude. The lack of a significant (negative) correlation in musicians suggests that there is no simple trade-off between modality-specific attentional capacity or strategy. 


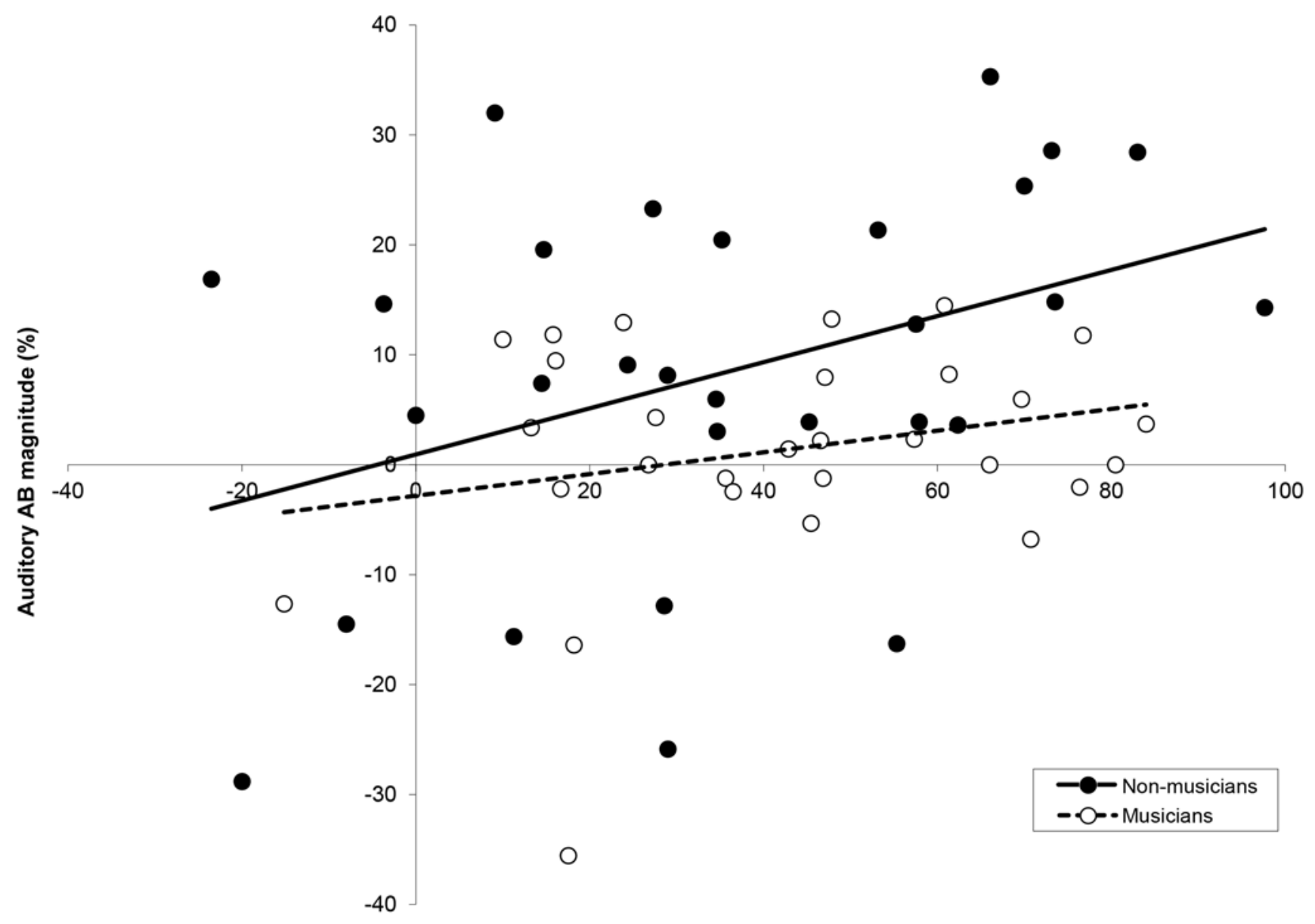

Visual $A B$ magnitude (\%)

Fig 6. $A B$ magnitudes in Experiment 1 and 2. The correlation between individual $A B$ magnitudes in the visual modality (Experiment 2 ) versus auditory modality (Experiment 1). Large visual blinkers tend to be large auditory blinkers, unless one is a musician.

\section{General Discussion}

Even though the $\mathrm{AB}$ has long been considered as a fundamental bottleneck to become consciously aware of relevant information, several recent studies have argued against the existence of a hard-wired limitation, showing that experimental manipulations can strongly affect $A B$ magnitude [38-40,46,53-62]. In addition, large individual differences in AB magnitude have been reported (e.g., [25]). If temporal attention is indeed under strategic control that varies between individuals, an important question is how general such a processing strategy is within individuals.

To our knowledge, this is the first study to show that the auditory $\mathrm{AB}$ in musicians is both delayed and attenuated using non-musical stimuli, possibly reflecting a more efficient attentional distribution $[25,39,44,46,47,54,63]$. In contrast, the distribution of attention within the visual modality seems less optimal, evidenced by a larger visual $A B$ and more lag- 1 sparing in musicians, relative to performance in participants without musical background. The domaingeneral but modality-specific effect of formal musical training is congruent with a study on mental imagery, which reported that musicians outperformed non-musicians on a nonmusical auditory imagery task, but not on a visual imagery task [8]. 
In line with previous results [35], non-musicians with a large auditory $\mathrm{AB}$ also showed a large visual $\mathrm{AB}$, suggesting that a similar target selection strategy was used for both modalities. Indeed individual auditory $\mathrm{T} 1$ performance correlated with visual $\mathrm{T} 1$ performance, for both groups. However, neither a positive nor a negative correlation was found between the musicians' auditory and visual $\mathrm{AB}$ magnitudes, suggesting that at least in musicians, attentional restrictions within each modality are completely separate.

Whereas we previously reported that some individuals (non-blinkers) can show a significant auditory $\mathrm{AB}$ but little or no visual $\mathrm{AB}$ [34], the present study demonstrates that the opposite result can also be found: A given individual can show substantial attentional restrictions within the visual modality but reduced restrictions within the auditory modality. This contrasting pattern of results provides further evidence that a major source of attentional restriction as reflected in the $\mathrm{AB}$ lies in modality-specific, independent sensory systems rather than a central amodal system.

While it must be noted that attentional selection of multiple targets is likely to be restricted not only by modality-specific but also by central limitations [64-68], our current findings suggest that musical training seems particularly beneficial within the auditory modality rather than at a central amodal level. The particular expertise that musicians have gained through years of training may have optimized the formation as well as handling of auditory representations. This may allow for a more optimal timing of attentional allocation to auditory targets [58] similar to the more optimal deployment of attention for visual targets that has been observed in non-blinkers [25]. Though we did not find a negative correlation between visual and auditory AB magnitudes in musicians, their relative expertise for auditory information may have caused them to put relatively more effort in the identification of visual targets. The musicians' increased lag-1 sparing as well as $\mathrm{AB}$ magnitude in our visual $\mathrm{AB}$ task provides support for this idea, in line with the overinvestment hypothesis that we and others have previously suggested $[46,47,53,63]$.

Although we find it plausible to assume that musical training caused the described changes in attentional restrictions, additional research is required in order to empirically validate this assumption. If musical training really causes a decrease in auditory $A B$ magnitude, it would be interesting to determine which aspects of the training actually contribute to the effect on the one hand, and which specific cognitive processes that are known to play a role in the $\mathrm{AB}$ are affected on the other hand.

\section{Conclusions}

We conclude that people who are musically trained show an attenuated and delayed $\mathrm{AB}$ when required to identify two auditory targets amongst a stream of non-targets, possibly reflecting a more efficient allocation of attention. In contrast, the distribution of attention within the visual modality seems less optimal, evidenced by a larger visual $\mathrm{AB}$ and more lag- 1 sparing in musicians, relative to performance in participants without musical background.

Although in the current study non-musicians with a relatively large auditory $\mathrm{AB}$ also tend to show a relatively large visual $\mathrm{AB}$ (also see [35]), neither a positive nor negative correlation was found for the musicians. The results reported here fit with the idea that a major source of attentional restriction as reflected in the $\mathrm{AB}$ lies in modality-specific, independent sensory systems rather than a central amodal system $[35,65,69,70]$. This does not mean that attention is not limited at a central level. For instance, the positive correlation between visual and auditory T1 accuracy that we generally observed in both musicians and non-musicians may well arise from individual restrictions at a more central, amodal level.

In addition, the results show that formal musical training can indeed have domain-general (i.e., not specific to the perception of music) beneficial effects, but that at least some of these 
effects are limited to the auditory modality and do not carry over to the visual modality. Taken together, the findings demonstrate that individual differences in $\mathrm{AB}$ magnitude can provide important information about the modular structure of human cognition.

\section{Supporting Information}

S1 Dataset.

(CSV)

\section{Acknowledgments}

We thank two anonymous reviewers for their helpful comments on an earlier version of this article. Correspondence concerning this article should be addressed to Sander Martens, Neuroimaging Center, Antonius Deusinglaan 2, 9713 AW Groningen, the Netherlands. E-mail: s.martens@umcg.nl.

\section{Author Contributions}

Conceived and designed the experiments: SM SMW MD MdV HGOMS. Performed the experiments: MD MdV. Analyzed the data: SMW MD MdV. Wrote the paper: SM SMW MD MdV HGOMS.

\section{References}

1. Hannon EE, Trainor LJ. Music acquisition: effects of enculturation and formal training on development. Trends Cogn Sci 2007; 11: 466-472. PMID: 17981074

2. Fujioka T, Trainor LJ, Ross B, Kakigi R, Pantev C. Automatic encoding of polyphonic melodies in musicians and nonmusicians. J Cogn Neurosci 2005; 17: 1578-1592. PMID: 16269098

3. Pantev C, Roberts LE, Schulz M, Engelien A, Ross B. Timbre-specific enhancement of auditory cortical representations in musicians. Neuroreport 2001; 12: 169-174. PMID: 11201080

4. Peretz I, Zatorre RJ. Brain organization for music processing. Annu Rev Psychol 2005; 56: 89-114. PMID: 15709930

5. Schneider P, Sluming V, Roberts N, Scherg M, Goebel R, Specht HJ, et al. Structural and functional asymmetry of lateral Heschl's gyrus reflects pitch perception preference. Nat Neurosci 2005; 8 : 1241-1247. PMID: 16116442

6. Shahin A, Bosnyak DJ, Trainor LJ, Roberts LE. Enhancement of neuroplastic P2 and N1c auditory evoked potentials in musicians. J Neurosci 2003; 23: 5545-5552. PMID: 12843255

7. Wong PCM, Skoe E, Russo NM, Dees T, Kraus N. Musical experience shapes human brainstem encoding of linguistic pitch patterns. Nat Neurosci 2007; 10: 420-422. PMID: 17351633

8. Aleman A, Nieuwenstein MR, Böcker KB, de Haan EH. Music training and mental imagery ability. Neuropsychologia 2000; 38: 1664-1668. PMID: 11074089

9. Gaser C, Schlaug G. Brain structures differ between musicians and non-musicians. J Neurosci 2003; 23: 9240-9245. PMID: 14534258

10. Pantev C, Oostenveld R, Engelien A, Ross B, Roberts LE, Hoke M. Increased auditory cortical representation in musicians. Nature 1998; 392: 811-814. PMID: 9572139

11. Koelsch $S$, Schröger E, Tervaniemi M. Superior pre-attentive auditory processing in musicians. Neuroreport 1999; 10: 1309-1313. PMID: 10363945

12. Peretz I, Coltheart M. Modularity of music processing. Nat Neurosci 2003; 6: 688-691. PMID: 12830160

13. Anvari SH, Trainor LJ, Woodside J, Levy BA. Relations among musical skills, phonological processing, and early reading ability in preschool children. J Exp Child Psychol 2002; 83: 111-130. PMID: 12408958

14. Schellenberg EG. Music and Cognitive Abilities. Curr Dir Psychol Sci 2005; 14: 317-320.

15. Schellenberg EG. Music lessons enhance IQ. Psychol Sci 2004; 15: 511-514. PMID: 15270994 
16. Gibson C, Folley BS, Park S. Enhanced divergent thinking and creativity in musicians: a behavioral and near-infrared spectroscopy study. Brain Cogn 2009; 69: 162-169. doi: 10.1016/j.bandc.2008.07.009 PMID: 18723261

17. Fujioka T, Ross B, Kakigi R, Pantev C, Trainor LJ. One year of musical training affects development of auditory cortical-evoked fields in young children. Brain 2006; 129: 2593-2608. PMID: 16959812

18. Moreno S, Besson M. Musical training and language-related brain electrical activity in children. Psychophysiology 2006; 43: 287-291. PMID: 16805867

19. Pallesen KJ, Brattico E, Bailey CJ, Korvenoja A, Koivisto J, Gjedde A, et al. Cognitive control in auditory working memory is enhanced in musicians. PLoS One 2010; 5: e11120. doi: 10.1371/journal.pone. 0011120 PMID: 20559545

20. Zuk J, Benjamin C, Kenyon A, Gaab N. Behavioral and Neural Correlates of Executive Functioning in Musicians and Non-Musicians. PLoS One 2014; 9: e99868. doi: 10.1371/journal.pone.0099868 PMID: 24937544

21. Martens S, Wyble B. The attentional blink: past, present, and future of a blind spot in perceptual awareness. Neurosci Biobehav Rev 2010; 34: 947-957. doi: 10.1016/j.neubiorev.2009.12.005 PMID: 20025902

22. Duncan J, Ward R, Shapiro K. Direct measurement of attentional dwell time in human vision. Nature 1994; 369: 313-315. PMID: 8183369

23. Dux PE, Marois R. The attentional blink: a review of data and theory. Atten Percept Psychophys 2009; 71: 1683-1700. doi: 10.3758/APP.71.8.1683 PMID: 19933555

24. Shapiro KL, Raymond JE, Arnell KM. The attentional blink. Trends Cogn Sci 1997; 1: 291-296. doi: 10. 1016/S1364-6613(97)01094-2 PMID: 21223931

25. Martens S, Munneke J, Smid H, Johnson A. Quick minds don't blink: electrophysiological correlates of individual differences in attentional selection. J Cogn Neurosci 2006; 18: 1423-1438. PMID: 16989545

26. Slagter HA, Johnstone T, Beets IAM, Davidson RJ. Neural competition for conscious representation across time: an fMRI study. PLoS One 2010; 5: e10556. doi: 10.1371/journal.pone.0010556 PMID: 20479939

27. Colzato L, Spapé M, Pannebakker M, Hommel B. Working memory and the attentional blink: Blink size is predicted by individual differences in operation span. Psychon Bull Rev 2007; 14: 1051-1057. PMID: 18229474

28. Colzato L, Hommel B, Shapiro K. Religion and the attentional blink: Depth of faith predicts depth of the blink. Cognition 2010; 45: 627-646.

29. Dale G, Arnell K. Individual differences in dispositional focus of attention predict attentional blink magnitude. Attention, Perception, Psychophys 2010; 72: 602-606. doi: 10.3758/APP.72.3.602 PMID: 20348566

30. Dux PE, Marois R. Distractor inhibition predicts individual differences in the attentional blink. PLoS One 2008; 3: e3330. doi: 10.1371/journal.pone.0003330 PMID: 18833325

31. Maclean $\mathrm{MH}$, Arnell KM. Personality predicts temporal attention costs in the attentional blink paradigm. Psychon Bull Rev 2010; 17: 556-562. doi: 10.3758/PBR.17.4.556 PMID: 20702877

32. Martens S, Dun M, Wyble B, Potter MC. A quick mind with letters can be a slow mind with natural scenes: Individual differences in attentional selection. PLoS One 2010; 5: e13562. doi: 10.1371/journal. pone.0013562 PMID: 21048954

33. Martens $\mathrm{S}$, Johnson A. Working memory capacity, intelligence, and the magnitude of the attentional blink revisited. Exp Brain Res 2009; 192: 43-52. doi: 10.1007/s00221-008-1551-1 PMID: 18762929

34. Martens S, Johnson A, Bolle M, Borst J. A quick visual mind can be a slow auditory mind. Individual differences in attentional selection across modalities. Exp Psychol 2009; 56: 33-40. doi: 10.1027/16183169.56.1.33 PMID: 19261576

35. Martens S, Kandula M, Duncan J. Restricted attentional capacity within but not between sensory modalities: an individual differences approach. PLoS One 2010; 5: e15280. doi: 10.1371/journal.pone. 0015280 PMID: 21151865

36. Martens S, Korucuoglu O, Smid HGOM, Nieuwenstein MR. Quick minds slowed down: effects of rotation and stimulus category on the attentional blink. PLoS One 2010; 5: e13509. doi: 10.1371/journal. pone.0013509 PMID: 20975838

37. Martens S, Valchev N. Individual differences in the attentional blink. The important role of irrelevant information. Exp Psychol 2009; 56: 18-26. doi: 10.1027/1618-3169.56.1.18 PMID: 19261574

38. Slagter HA, Lutz A, Greischar LL, Francis AD, Nieuwenhuis S, Davis JM, et al. Mental training affects distribution of limited brain resources. PLoS Biol 2007; 5: e138. PMID: 17488185 
39. Taatgen NA, Juvina I, Schipper M, Borst JP, Martens S. Too much control can hurt: A threaded cognition model of the attentional blink. Cogn Psychol 2009; 59: 1-29. doi: 10.1016/j.cogpsych.2008.12.002 PMID: 19217086

40. Green CS, Bavelier D. Action video game modifies visual selective attention. Nature 2003; 423: 534-537. PMID: 12774121

41. Colzato LS, Bajo MT, van den Wildenberg W, Paolieri D, Nieuwenhuis S, La Heij W, et al. How does bilingualism improve executive control? A comparison of active and reactive inhibition mechanisms. J Exp Psychol Learn Mem Cogn 2008; 34: 302-312. doi: 10.1037/0278-7393.34.2.302 PMID: 18315407

42. Arnell KM, Stokes KA, MacLean MH, Gicante C. Executive control processes of working memory predict attentional blink magnitude over and above storage capacity. Psychol Res 2010; 74: 1-11. doi: 10. 1007/s00426-008-0200-4 PMID: 19084999

43. Arnell KM, Stubitz SM. Attentional blink magnitude is predicted by the ability to keep irrelevant material out of working memory. Psychol Res 2010; 74: 457-467. doi: 10.1007/s00426-009-0265-8 PMID: 19937451

44. Willems C, Wierda SM, van Viegen E, Martens S. Individual differences in the attentional blink: the temporal profile of blinkers and non-blinkers. PLoS One 2013; 8: e66185. doi: 10.1371/journal.pone. 0066185 PMID: 23755299

45. Schneider W, Eschman A, Zuccolotto A. E-prime user's guide. Pittsburgh: Psychology Software Tools; 2002. doi: 10.3758/s13428-012-0302-1 PMID: 23292569

46. Wierda SM, van Rijn H, Taatgen NA, Martens S. Distracting the mind improves performance: an ERP Study. PLoS One 2010; 5: e15024. doi: 10.1371/journal.pone.0015024 PMID: 21124833

47. Wierda SM, van Rijn H, Taatgen NA, Martens S. Pupil dilation deconvolution reveals the dynamics of attention at high temporal resolution. Proc Natl Acad Sci U S A 2012; 109: 8456-8460. doi: 10.1073/ pnas.1201858109 PMID: 22586101

48. Baayen RH, Davidson DJ, Bates DM. Mixed-effects modeling with crossed random effects for subjects and items. J Mem Lang 2008; 59: 390-412.

49. Barr DJ. Analyzing "visual world" eyetracking data using multilevel logistic regression. J Mem Lang 2008; 59: 457-474.

50. Kliegl R, Masson MEJ, Richter EM. A linear mixed model analysis of masked repetition priming. Vis cogn 2010; 18: 655-681.

51. Bates D, Maechler M, Dai B. Ime4: Linear mixedeffects models using S4 classes. 2008.

52. Potter MC, Staub A, O'Connor DH. The time course of competition for attention: Attention is initially labile. J Exp Psychol Hum Percept Perform 2002; 28: 1149-1162. PMID: 12421061

53. Olivers CNL, Nieuwenhuis S. The beneficial effects of additional task load, positive affect, and instruction on the attentional blink. J Exp Psychol Hum Percept Perform 2006; 32: 364-379. PMID: 16634676

54. Olivers $\mathrm{C}$, Nieuwenhuis $\mathrm{S}$. The beneficial effect of concurrent task-irrelevant mental activity on temporal attention. Psychol Sci 2005; 16: 265-269. PMID: 15828972

55. Arend I, Johnston S, Shapiro K. Task-irrelevant visual motion and flicker attenuate the attentional blink. Psychon Bull Rev 2006; 13: 600-607. PMID: 17201358

56. Olivers CNL, Meeter M. A boost and bounce theory of temporal attention. Psychol Rev 2008; 115 : 836-863. doi: 10.1037/a0013395 PMID: 18954206

57. Ferlazzo F, Lucido S, Di Nocera F, Fagioli S, Sdoia S. Switching Between Goals Mediates the Attentional Blink Effect. Exp Psychol (formerly "Zeitschrift für Exp Psychol 2007; 54: 89-98.

58. Martens S, Johnson A. Timing attention: cuing target onset interval attenuates the attentional blink. Mem Cognit 2005; 33: 234-240. PMID: 16028578

59. Nieuwenstein MR, Chun MM, van der Lubbe RHJ, Hooge ITC. Delayed attentional engagement in the attentional blink. J Exp Psychol Hum Percept Perform 2005; 31: 1463-1475. PMID: 16366802

60. Potter MC, Nieuwenstein M, Strohminger N. Whole Report versus Partial Report in RSVP Sentences. J Mem Lang 2008; 58: 907-915. PMID: 18568099

61. Tang MF, Badcock DR, Visser TAW. Training and the attentional blink: limits overcome or expectations raised? Psychon Bull Rev 2014; 21: 406-411. doi: 10.3758/s13423-013-0491-3 PMID: 23884691

62. Choi H, Chang L- H, Shibata K, Sasaki Y, Watanabe T. Resetting capacity limitations revealed by longlasting elimination of attentional blink through training. Proc Natl Acad Sci U S A 2012; 109: 12242-12247. doi: 10.1073/pnas.1203972109 PMID: 22778408

63. Shapiro K, Schmitz F, Martens S, Hommel B, Schnitzler A. Resource sharing in the attentional blink. Neuroreport 2006; 17: 163-166. PMID: 16407764 
64. Vachon F, Tremblay S. Modality-specific and amodal sources of interference in the attentional blink. Percept Psychophys 2008; 70: 1000-1015. PMID: 18717386

65. Duncan J, Martens S, Ward R. Restricted attentional capacity within but not between sensory modalities. Nature 1997; 387: 808-810. PMID: 9194561

66. Arnell KM, Jenkins R. Revisiting within-modality and cross-modality attentional blinks: effects of targetdistractor similarity. Percept Psychophys 2004; 66: 1147-1161. PMID: 15751472

67. Arnell KM, Duncan J. Separate and shared sources of dual-task cost in stimulus identification and response selection. Cogn Psychol 2002; 44: 105-147. PMID: 11863322

68. Jolicoeur P, Dell'Acqua R. The demonstration of short-term consolidation. Cogn Psychol 1998; 36 : 138-202. PMID: 9721199

69. Soto-Faraco S, Spence C. Modality-specific auditory and visual temporal processing deficits. Q J Exp Psychol A 2002; 55: 23-40. PMID: 11873849

70. Hein G, Parr A, Duncan J. Within-modality and cross-modality attentional blinks in a simple discrimination task. Percept Psychophys 2006; 68: 54-61. PMID: 16617829 